\title{
Investigating the relationship between how a chief complaint is expressed and the patient workflow
}

\author{
Sajjad Ahmadi', Niloufar Pouresmaeil', Farima Najjarian', Samad Shams Vahdati', Maryam Rahimpour \\ Asenjan², Hamid Reza Morteza Bagi ${ }^{1 *}$ \\ 'Emergency Medicine Research Team, Faculty of Medicine, Tabriz University of Medical Sciences, Tabriz, Iran \\ ${ }^{2}$ Emergency Medicine Research Team, Faculty of Nursing and Midwifery, Tabriz University of Medical Sciences, Tabriz, Iran
}

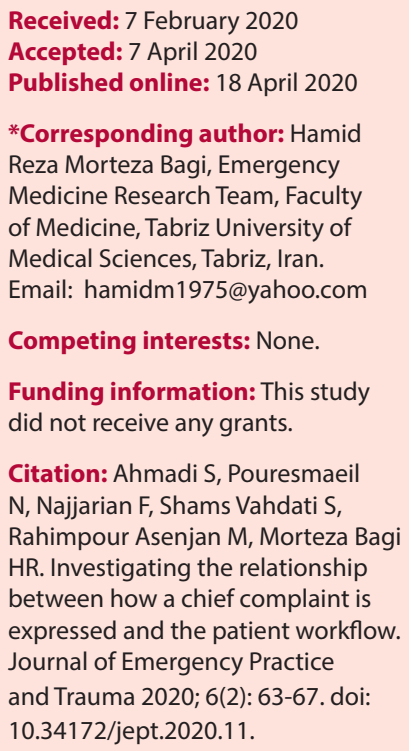

\begin{abstract}
Objective: Emergency departments and hospital emergency departments are important due to their critical role in providing urgent medical care to patients in dire need of medical interventions. Checking bottlenecks in new conditions and planning to reduce bed occupancy and hospitalization is needed. The purpose of this study is to investigate the relationship between the patient's chief complaint and their departure to the emergency room.

Methods: From non-traumatic patients referred to the emergency department of Imam Reza Hospital during 2018, about 57000 patients were selected and enrolled in the study. Then, age, sex, initial diagnosis, time of the final decision, and time of departure from the emergency department as well as hospitalization ward were included in the checklist. Patients whose documentation was incomplete were excluded. Data were entered into SPSS software version 15.0 and descriptive statistics (normal distribution, average of time, minimum time and maximum time, confidence interval, mode, and median, etc) were used for descriptive analysis and linear regression was used to analyze the correlation among findings.

Results: There was a significant relationship between chief complaint and the length of stay in the emergency department $(P=0.046)$ and patients with dyspnea due to heart disease, bloody vomit, bloody stool, constipation, jaundice, anemia, decreased level of consciousness, diabetes, complications of diabetes, shortness of breath and kidney injury stayed longer in the emergency room compared to other complaints.

Conclusion: The patient's manner of expressing and chief complaint has an impact on the length of time they wait to leave the emergency room. Also, most patients with problems related to internal medicine have the longest time in the emergency room; in particular gastrointestinal patients have the longest stay in the emergency room.

Keywords: Demography, Initial diagnosis, Chief complaint, Emergency medicine
\end{abstract}

\section{Introduction}

Emergency departments and hospital emergency departments are one of the indispensable pillars of public hospitals in a country due to their critical role in providing urgent medical care to patients in dire need of prompt treatment at all times of day and night $(1,2)$. The efficacy of the emergency department is evaluated by waiting times. The speed of service delivery in health centers, especially in emergencies, is particularly important in reducing the mortality and morbidity of any disability (3). Patients' waiting time may also lead to the formation of negative attitudes towards the hospital and care providers $(2,4)$. The duration of stay is influenced by individual characteristics such as age, sex, disease status, patient management style, and organizational and management characteristics of the hospital (4). Also, in the three Canadian, the US, and the UK countries, the percentage of those who stayed in the emergency room for less than 4 hours was $76 \%, 72 \%$, and $96 \%$, respectively (5). Approaches to admission and discharge, quality and quantity of communication and interdepartmental collaborations, including the collaborations with other medical departments in admission, and support for important factors affecting the duration of stay have been demonstrated in various studies $(1,6,7)$. The patient process in the emergency department includes all the steps that are taken from the patient's arrival to the emergency department to the final diagnosis and management. This 
determines the duration of time the patient is in the emergency department. All departments must work as part of a team, and if one of them is in trouble and unable to function effectively, bottlenecks will develop (8).

In previous studies workflows in the emergency department of Imam Reza hospital, as the general center in northwestern and East Azarbaijan province, with the focus on bottlenecks and hospital admissions as well as management were conducted $(9,10)$, but considering that these studies are not up to date and due to the Health Transformation Plan implemented by the Ministry of Health, the hospital workflow has been changed and the state hospitals have become very crowded. In this regard, the bottlenecks have been changed. Therefore, there is a need to review bottlenecks in new conditions and plan to reduce bed occupancy and hospitalization. The purpose of this study is to investigate the relationship between the patients' chief complaint and their departure to the emergency room.

\section{Methods}

This is a retrospective, cross-sectional descriptive study. Non-traumatic patients who referred to the emergency department of Imam Reza hospital during 2018 were selected and included in the study. Patients whose documentation was incomplete were excluded.

All patients who had any medical chief complain entered the study, patients who had trauma or his document were not clear were excluded. Age, sex, chief complaint and time of final decision and time of departure from the emergency department as well as hospitalization to the ward were included in the checklist.

The obtained data were entered into IBM SPSS software version 15.0 and analyzed with the significance level below 0.05 . The intraclass correlation coefficient was used to evaluate the agreement between the evaluators at both time points. Pearson's regression was used to examine the relationship between the collected data. The significance level was considered as $P<0.05$.

\section{Results}

In this study, all non-traumatic patients referring to Imam Reza hospital in the year 2018 were 57000 which did not follow a normal distribution based on KolmogorovSmirnov analysis $(P<0.001)$. The mean age of patients was $53.34 \pm 20.38$ years with a median of 53.00 years and mode was 40 years. The minimum age was 1 year and the maximum was 116 years. In terms of sex, 31588 patients (55.6\%) were males and 25250 were females (44.4\%). The most common complaints of patients ware weakness, abdominal pain, headache and dizziness (Table 1).

Over $46 \%$ of patients were discharged from the emergency department and $17 \%$ of patients were admitted to hospital wards. In addition, $32 \%$ of patients had either escaped or left the emergency department against medical advice,
Table 1. Frequency of patients' chief complaints

\begin{tabular}{|c|c|c|}
\hline & Frequency & Percent \\
\hline Without chief complaint & 528 & 0.9 \\
\hline Kidney problem & 1159 & 2.0 \\
\hline Headache and vertigo & 5693 & 10.0 \\
\hline Flank pain & 2816 & 5.0 \\
\hline Weakness & 13951 & 24.5 \\
\hline Low back pain & 1912 & 3.4 \\
\hline Fever and chill & 608 & 1.1 \\
\hline Dyspnea & 4017 & 7.1 \\
\hline DM with complication & 921 & 1.6 \\
\hline Epigastric pain & 555 & 1.0 \\
\hline Loss of consciousness & 1768 & 3.1 \\
\hline Urination retention & 613 & 1.1 \\
\hline One limb swelling & 715 & 1.3 \\
\hline Anemia & 610 & 1.1 \\
\hline Chest pain & 267 & 0.5 \\
\hline Icterus & 62 & 0.1 \\
\hline FAST sign & 1326 & 2.3 \\
\hline Abdominal pain & 9824 & 17.3 \\
\hline Seizure & 1124 & 2.0 \\
\hline High blood pressure & 691 & 1.2 \\
\hline Known intestinal cancer & 9 & 0.0 \\
\hline Drug toxicity or overdose & 101 & 0.2 \\
\hline Bloody stool & 607 & 1.1 \\
\hline Ear foreign body & 25 & 0.0 \\
\hline Obstipation and constipation & 53 & 0.1 \\
\hline Abdominal fluid tap & 386 & 0.7 \\
\hline Blood transfusion & 67 & 0.1 \\
\hline Esophageal foreign body & 178 & 0.3 \\
\hline Blood pressure drop & 263 & 0.5 \\
\hline Bloody vomit & 128 & 0.2 \\
\hline Heart failure and dyspnea & 9 & 0.0 \\
\hline Right upper stomach ache & 27 & 0.0 \\
\hline Sore throat & 2 & 0.0 \\
\hline Food poisoning & 16 & 0.0 \\
\hline Gastric cancer & 10 & 0.0 \\
\hline Carbon monoxide poisoning & 16 & 0.0 \\
\hline Esophagus cancer & 6 & 0.0 \\
\hline Undefined chief complaint & 5775 & 10.2 \\
\hline
\end{tabular}

or referred to a designated hospital by personal vehicle. Considering that all these three types were ultimately recorded as discharge against medical advice, it was not possible to distinguish them (Figure 1).

Among these patients, 5119 (9\%) received outpatient prescriptions without staying in the emergency department. This indicates that the patient was only checked by a physician and left. Also, 35785 patients 


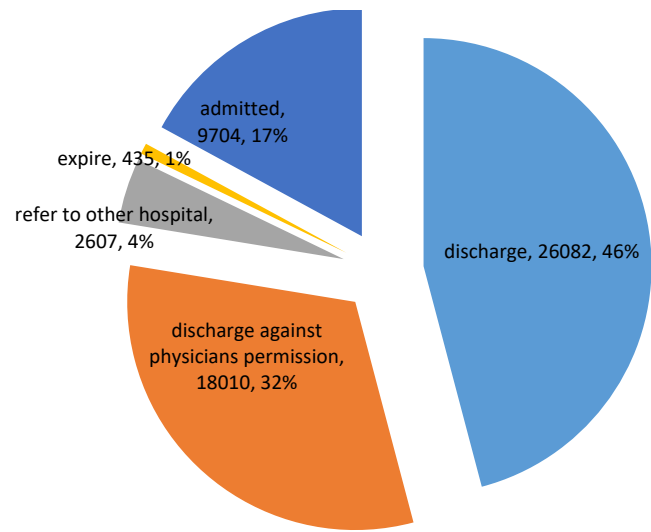

Figure 1. Frequency of patients' final fate.

(63\%), left within 6 hours and 8555 patients (15.1\%), left the emergency room within 6 to 12 hours. On the whole, 44340 patients, excluding single-visit patients, accounted for $78 \%$ of patients who left under 12 hours. With regard to single-visit patients, $87 \%$ of non-traumatic patients ( $\mathrm{n}=$ 49459), were discharged under 12 hours.

There was a significant relationship between the chief complaint and the duration of stay in the emergency department $(P=0.046)$. Patients with dyspnea due to heart disease, bloody vomit, bloody stool, constipation, jaundice, anemia, decreased consciousness levels, diabetes, diabetes complications, shortness of breath and kidney discomfort stayed longer in the emergency room compared to other complaints or average staying time (Figure 2).

\section{Discussion}

In the study by Hosseini et al, the mean age of patients was about 40 years, similar to the study by Cardin et al in traumatic patients admitted in the emergency room $(11,12)$. In the current study, 31588 patients $(55.6 \%)$ were males and 25250 were females (44.4\%). Hosseini et al indicated that the majority of patients were men and only $20 \%$ of patients were women (11). The most common complaints in patients were weakness, abdominal pain, headache and dizziness.

In our study, $46 \%$ of patients were discharged from the emergency department and $17 \%$ were admitted to hospital wards. Also, 32\% of patients had either escaped or left the emergency department against medical advice, or referred to a designated hospital by personal vehicle. Considering that all these three types were ultimately recorded as discharge against medical advice, it was not possible to differentiate them.

In our investigation, 5119 (9\%) patients received outpatient prescriptions without staying in the emergency ward indicating that the patient was only checked by a physician and left the ward. Besides, 35785 patients (63\%) left within 6 hours and 8555 patients (15.1\%) left the emergency room within 6 to 12 hours. In general, 44340 patients, excluding single-visit patients, accounted for $78 \%$ of patients who left under 12 hours. Taking into account only the single-visits $(\mathrm{n}=49459 ; 87 \%)$, these patients left the emergency room under 12 hours. However, in a study conducted by Rahi et al, comparing the stopping time of patients admitted to the emergency department before and after the establishment of an emergency medicine specialist, it was shown that the average stopping time of patients admitted before the presence of the emergency medicine specialist was 4 hours and the time after his presence was 3 hours (13). It is important to mention that the duration of stay in Imam Reza emergency room was higher than the length of stay in the mentioned study. According to the guidelines of the Ministry of Health and Medical Education, patients stay in the emergency department for a maximum of 6 hours. However, a significant number of patients remained in

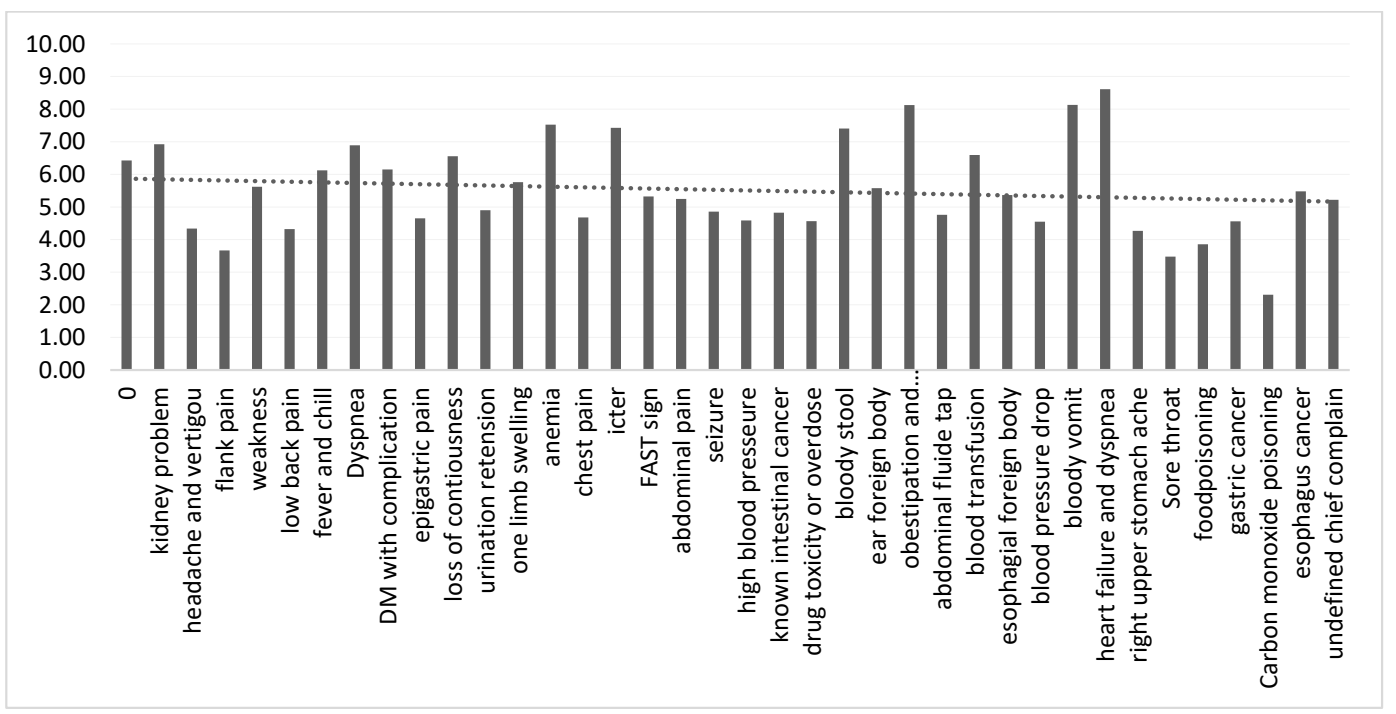

Figure 2. The average stay of the patients based on the main complaint. 
the emergency department for a long time due to the high number of patients referring to specialties not related to the center, the overcrowding of wards, and the significant number of unnecessary and non-emergency paraclinical requests. The presence of the emergency specialists plays an important role in improving services and patient satisfaction through the management of noted problems (14). The study of Hosseini et al found that the most important reasons for the decline in bed occupancy rate, daily bed occupancy and increased bed activity were the timely decision making for patients after the presence of an emergency medicine specialist (11).

In our study, on the whole, 44340 patients (78\%), excluding single-visit patients, left under 12 hours. Also, 49459 (87\%) patients, including single-visit patients, left the emergency room under 12 hours. Therefore, patients' stay and its duration are important indicators in evaluating the quality of emergency services. In a study conducted in hospital emergency departments at the University of California in order to assess patients' waiting time, results showed that patients waited for an average of 56 minutes for a physician and $42 \%$ of patients had a wait time more than 60 minutes (15).

In a study conducted by Amiri et al in Imam Reza hospital, despite the hurdles such as working with a new emergency medicine team and lacking sufficient workforce to manage the ward, patients were cared within a few minutes. Besides, the first visit time was brief and all patients were visited by residents and medical teachers (9). Previous researches have been conducted on the workflows in the emergency department of Imam Reza hospital with a focus on bottlenecks and hospital admissions rate, patients and management $(9,10)$. Findings revealed that there was a significant relationship between the chief complaint and the duration of stay in the emergency department $(P=$ 0.046). In addition, patients with dyspnea due to heart disease, bloody vomit, bloody stool, constipation, jaundice, anemia, decreased consciousness levels, diabetes, diabetes complications, shortness of breath, and kidney discomfort stayed longer in the emergency room compared to other complaints or average staying time.

\section{Conclusion}

How the patient present he/she chief complain has an impact on the length of time they wait to leave the emergency room, and most patients with problems related to internal medicine have the longest stay in the emergency room; in particular gastrointestinal patients have the longest stay in the emergency room.

\section{Authors' contribution}

Study design, HRMB; study conduct, SSV; data gathering, MRA and NP; supervision, SA; data analysis and writing, FN; critic, SSV.
Ethical issues

This study was approved by the Regional Ethic Committee of Research, Tabriz University of Medical Sciences, code no: IR.TBZMED.REC.1398.1101.

\section{References}

1. Ajami S, Ketabi S, Yarmohammadian MH, Bagherian H. Waiting time in emergency department by simulation. Stud Health Technol Inform 2011; 164: 196-200.

2. Zafarghandi M. University Strategies for Quality Improvement of Emergency System. Tehran: Proceedings of the First Congress on Quality Improvement in Emergency Management; 2011. [In Persian].

3. Khosravi S, Pouria-Mofrad A, Shamsipour M, Soorany $\mathrm{H}$. The effects of improvement plan on the emergency services in Kashani \& Hajar hospitals of Shahrekord. Journal of Shahrekord Uuniversity of Medical Sciences 2005; 7(2): 71-80. [In Persian].

4. Heydaranlou E, Khaghani Zadeh M, Ebadi A, Sirati Nir M, Aghdasi Mehr Abad N. A survey on implementation of FOCUS-PDCA on performance of Tabriz Shahid Mahalati emergency department. Journal of Military Medicine 2008; 10(4): 5-9. [In Persian].

5. Hay E, Bekerman L, Rosenberg G, Peled R. Quality assurance of nurse triage: consistency of results over three years. Am J Emerg Med 2001; 19(2): 113-7. doi: 10.1053/ ajem.2001.21317.

6. Asadi F. Improvement of Hospital Emergency Services by Quality Management System Approach. Tehran: Proceedings of the 4th International Congress on Quality; 2004. [In Persian].

7. Golaghaie F, Sarmadian H, Rafiie M, Nejat N. A study on waiting time and length of stay of attendants to emergency department of Vali-e-Asr Hospital, Arak-Iran. Journal of Arak University Medical Sciences 2008; 11(2): 74-83. [In Persian].

8. Ojaghi Haghighi SH, Shams Vahdati S, Sharifi Namin M, Sharifi Namin S, Habibollahi P, Mikaeilpour A. Comparing the patients' throughput in an emergency department in Tabriz city with international standards. Sakarya Tip Dergisi 2014; 4(3): 115-8. doi: 10.5505/ sakaryamj.2014.05025.

9. Amiri H, Shams Vahdati S, Ghodrati N, Taghizadueh A, Mahmoodivar S, et al. The length of waiting for first visit and disposition in emergency department in Tabriz Imam Reza hospital. Turk J Emerg Med 2008; 8(4): 160-3.

10. Ameri H, Adham D, Panahi M, Khalili Z, Fasihi A, Moravveji $\mathrm{M}$, et al. Predictors for duration of stay in hospitals. Journal of Health 2015; 6(3): 256-65. [In Persian].

11. Hosseini M, Shaker H, Basir Ghafouri H, Shokraneh F. Chronometric study of patients' workflow and effective factors on it in emergency department of 7th Tir Martyrs hospital of Tehran, Iran. Journal of Health Administration 2010; 13(40): 13-22. [In Persian].

12. Cardin S, Afilalo M, Lang E, Collet JP, Colacone A, Tselios $\mathrm{C}$, et al. Intervention to decrease emergency department crowding: does it have an effect on return visits and hospital readmissions? Ann Emerg Med 2003; 41(2): 17385. doi: 10.1067/mem.2003.50. 
13. Rahi F, Ale-Yasin A, Safdari S, Yaghoobi M. Audit time off Patient before and after the intervention in Emergency Medicine Department. First International \& 4th National Congress on health of Amin Hospital, Isfahan in 2011. Tehran, Iran: The Education \& Promotion; 2011. [In Persian].

14. Bidari A, Hatamabadi HR. The causes of hospitalization in patients over 24 hours in emergency department of Hazrat Rasoul Akram. Abstracts and presentations promoting quality in the first congress of Emergency management. Tehran: Iran Medical University; 2001. [In Persian].

15. Akbari ME. National Strategies for Quality Improvement in Emergency Management. Proceeding of the First National Conference on Quality Improvement in Emergency Medicine. Tehran: Tehran Medical Sciences University; 2002. [In Persian]. 